\title{
Manipulating Chemical Processes by Pseudosolid Spatial Limitation
}

\author{
Shin Yi, ${ }^{\dagger}$ Jia-Syuan Chen, ${ }^{\dagger}$ Chang-Ming Wang, and Wei-Ssu Liao*
}

Cite This: JACS Au 2021, 1, 1435-1444

Read Online

ABSTRACT: It is always preferred to perform chemical processes in liquid or gas phases because of the merits of operation convenience, reaction efficiency, and component homogeneity. However, tremendous efforts have to be made to purify the final product and minimize procedure losses unless a well-defined chemical mechanism is found. Herein, an unconventional chemical functioning system accommodating molecule-in-pseudosolid manipulation is reported. It entails the properties of enhanced molecular effective collision and directional guidance for delicate chemical reaction spatial controls. This design achieves facilitated rates on multicomponent chemical reactions with pros of unique

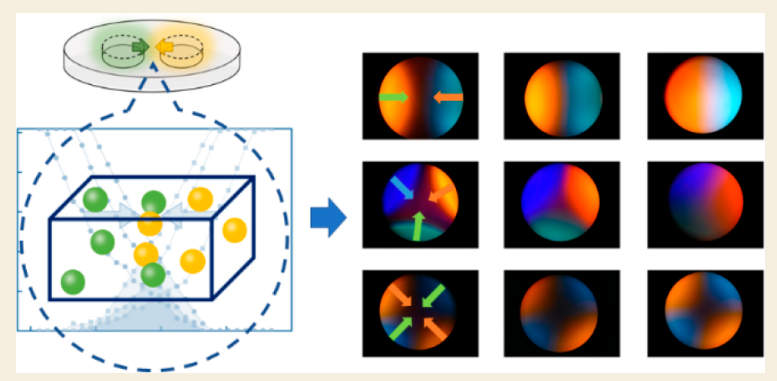
simultaneous final product separation through intrapseudosolid spatial limitation. Localized homogeneous component mixing, pronounced molecular collision, and pure product separation happening in this action surmount the obstacles of conventional chemical operation with a straightforward sketch. A path toward fine chemistry is therefore paved, where traditional thoughts on beneficial reaction environments may be reconsidered.

KEYWORDS: diffusion, spatial limitation, chemical reaction, separation, pseudosolid phase

\section{INTRODUCTION}

Chemical reactions are often performed in liquid or gas phases because the molecular mixing homogeneity is more controllable, the reaction rate is higher, and the operation of the experiment is more convenient. Because of the expected incomplete reactions or side product generation in a chemical process, additional posterior treatments, such as component separation and product purification, are always required. Tedious steps of the condition optimization and potential loss of the products, unfortunately, are therefore unavoidable unless a dedicated chemical mechanism is found. Unlike the other two commonly adapted matrices, solid medium is always less preferred for committing chemical reactions because the molecular moving speed is much slower and the species collision behavior is hard to control. These obvious drawbacks, therefore, highly restrict possibilities of utilizing a solid phase matrix for chemical operations not only in trace amount synthesis, but also for mass production. In this report, an unconventional interface approach for straightforward chemical reactions via the molecular behavior regulation in the pseudosolid phase is demonstrated. This strategy can overcome the aforementioned obstacles and achieve efficient chemical reactions with simultaneous product separation through a chromatography-like behavior in the matrix.

The main concept of this approach lies in a delicate control of spontaneous molecule net movements in solid-mimicking phases, i.e., the molecular diffusion commonly observed in daily life. The first scientific model to understand this behavior was provided by Thomas Graham in the 19th century, which describes the relationship between matter mass and its corresponding transportation in the gas phase. ${ }^{1,2}$ Adolf Fick thereafter depicted the first general diffusion effect illustration suitable for solid, liquid, and gas media via flux of molecules over a spot respect to time, which can be used not only in the steady-state condition but also in the non-steady-state condition. ${ }^{3,4}$ Integrating with Brownian motion theory, Stokes-Einstein's equation further reveals the relationship between the diffusion coefficient and other physical properties by a spherical particle in the liquid phase model. Important parameters, such as temperature, viscosity, and the particle radius, are included in the equation, which settles the modern atomistic theory of diffusion and can now be used for most circumstances under appropriate modifications. ${ }^{5-7}$ This basic equation representation is described as follows:

$$
D=\frac{k_{\mathrm{B}} T}{8 \pi \eta r^{3}}
$$

where $D$ is the diffusion constant, $k_{\mathrm{B}}$ is the Boltzmann's constant, $T$ is the absolute temperature, $\eta$ is the dynamic viscosity, and $r$ is the radius of a spherical particle.

To date, this description has been recognized as one of the most widely used formulas for investigating particle distribu-

Received: May 11, 2021

Published: August 3, 2021 
Scheme 1. Schematic Illustration of the Spatial Concentration Distribution Map of (A) Single-Source Ideal Solution Diffusion Behavior; (B) Double-Source Ideal Solution Diffusion Behavior; Corresponding 2D Form of (C) Single-Source and (D) Double-Source Concentration Distribution

(A)

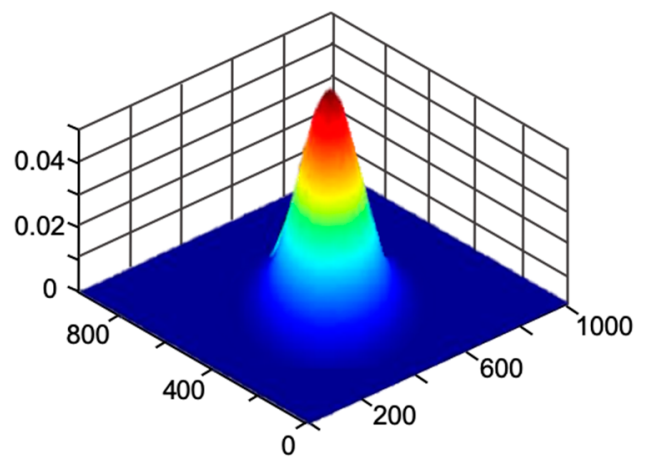

(C)

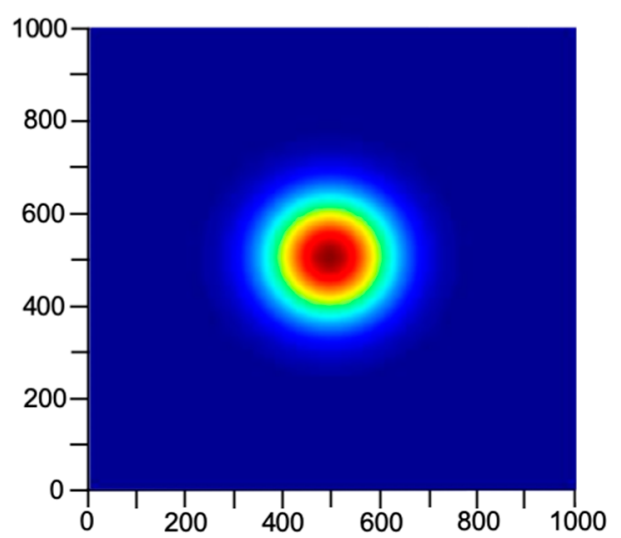

(B)

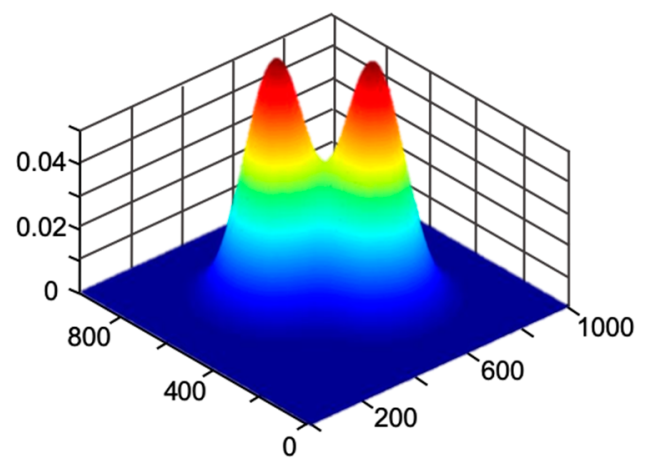

(D)

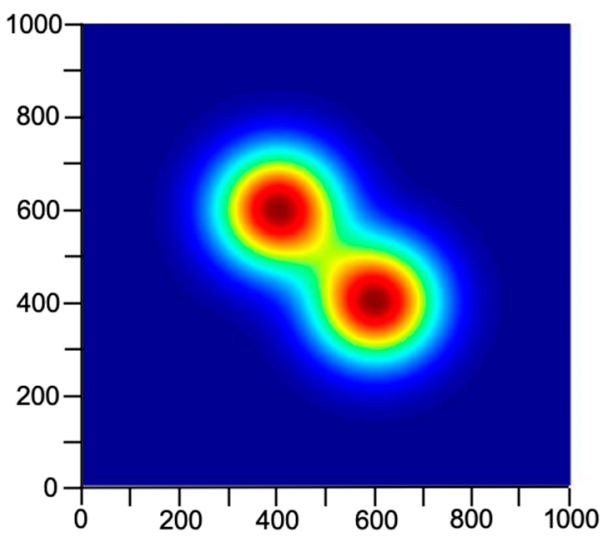

tion in a sophisticated matrix, which can provide suitable display of the matter moving behavior under divergent circumstances, such as minute sample extraction, interface molecular recognition, and cell biology metabolism. ${ }^{8-13}$

Although a variety of diffusion-based approaches have helped construct useful models for modern scientific and engineering issues, considerable attention has mainly been paid on liquid or gas environments. There is limited literature on solid-phase integrated diffusion systems, and related studies are constantly lacking mutual connections between fundamental understanding and practical application. However, molecular diffusion behavior in the solid-related phases plays a tremendous role in numerous experimental designs and may lead to unexpected effects if treated negligently. For example, various small molecules or organic solvents present obvious permeability toward cross-linked polydimethylsiloxane (PDMS) polymer conjugated networks (defined as pseudosolid in the study). The aforementioned phenomenon leads to significant experimental biases, including change of solution concentrations in microfluidic devices and obvious cell culture media composition disturbances. ${ }^{14,15}$ To avoid these undesirable interferences, we devoted huge efforts to reducing penetration of molecules into polymer networks, such as plasma treatment blocking, ${ }^{16}$ selective surface coating, ${ }^{17-20}$ and interspace material filling. ${ }^{21}$ Meanwhile, several correspondent experiments were testified and pointed to important factors involved in this matter transportation process, including molecular weight and size, ${ }^{22}$ analyte concentration, ${ }^{23}$ system temperature, ${ }^{23,24}$ and solvent solubility. ${ }^{16}$ Instead of prohibiting diffusion induced disadvantageous behavior, this unavoidable manner, on the contrary, may be considered as a useful tool for operations at the molecular level. For instance, PDMS can be used to absorb small hydrophobic molecules from an oil-water mixture to achieve target analyte extraction. ${ }^{25} \mathrm{~A}$ reversed operation was also demonstrated, where PDMS can act as a drug storage medium for subsequent diffusion-driven releasing processes. ${ }^{26,27}$ Despite the aforementioned possibilities, diffusion-based techniques in solid-related phases are still highly limited because of the challenges, such as effective tendency prediction and delicate spatial precision control, in these systems.

Standing on previous knowledge of molecular diffusion in a solid-related phase, the current design utilizes spontaneous solvent penetration as the driving force for the migration of reactants into pseudosolid polymer networks. This phenomenon creates a gradient molecular distribution within confined regions, and the resulting disparate reactant moving fronts are guided for directional overlapping to ensure specific chemical reaction occurrence at the joint diffusion boundary. Importantly, this interfacial process restricts the random movements of precursor molecules and reaction products as in gas or liquid phases, and the discrete separated component bands can be obtained directly at the end of operation. Taking advantage of this straightforward action and the delicate molecular movement controls, chemical reaction products can be simply extracted from band slices without the sophisticated purification commonly required in liquid/gas media-based methods.

\section{DISCUSSION}

When concentration variance happens between two spots, molecules tend to move from a more crowded environment to a less dense position to seek thermodynamic equilibrium, which plays an essential role in many physical, chemical, 
(A)
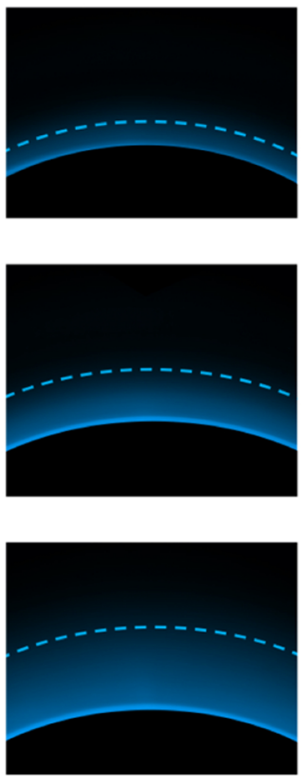

(B)

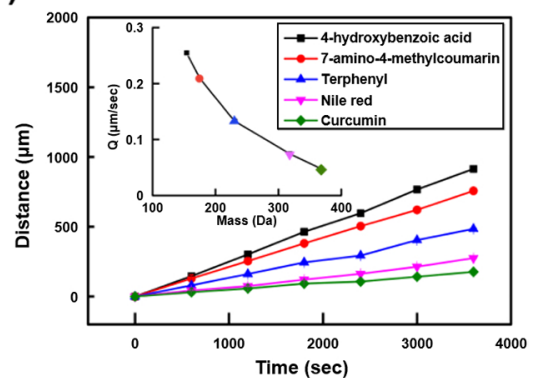

(D)

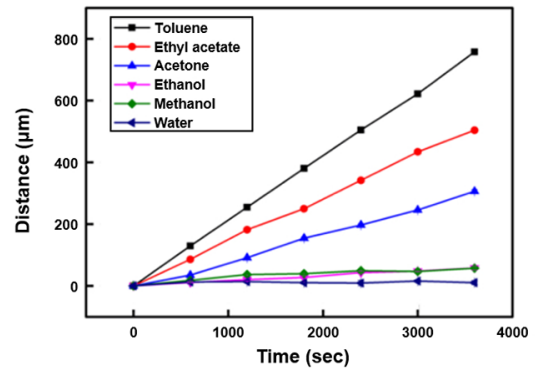

(C)

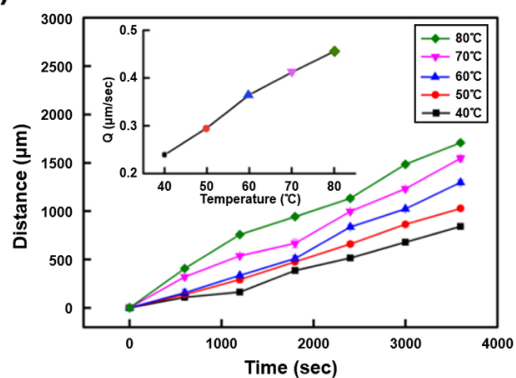

(E)

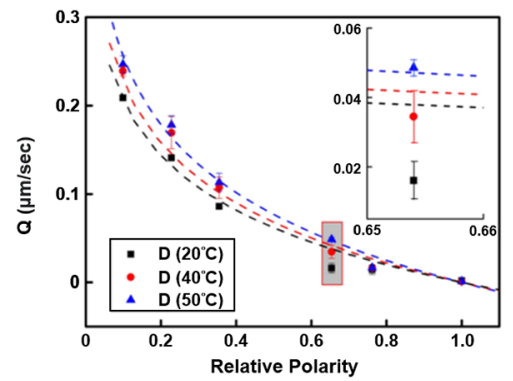

Figure 1. Molecular diffusion behavior and important affecting factors in the pseudosolid PDMS matrix. (A) Diffusion of fluorescent 7-amino-4methylcoumarin into PDMS after 10, 30, and 60 min of operation (from top to bottom) observed by an optical microscope. (B) Diffusion front moving distance of different analytes with time. Inset: change in $Q$ versus molecular mass. (C) Diffusion front moving distance of 7-amino-4methylcoumarin at different temperatures with time. Inset: change in $Q$ versus system temperature. (D) Diffusion front moving distance of 7amino-4-methylcoumarin with time when different carrying solvents are used. (E) Relationship between $Q$ and the relative polarity of carrying solvents when 7-amino-4-methylcoumarin is used as the diffusing analyte. The inset shows the enlarged part of the gray background area. The error bars indicate the standard deviation $(N=3)$.

biological, and engineering processes. ${ }^{28}$ As depicted in Scheme 1 , the spatial concentration distribution map of a single-source ideal solution based on Fick's diffusion law is demonstrated. The central part of this map represents the highest substance concentration, where the three-dimensional diffusion effect induces a final bell-shaped distribution within a period of time. This Gaussian-like concentration gradient distribution is attributed to the thermodynamics-driven molecular equilibrium seeking phenomenon between higher and lower substance levels in a matrix being preferentially terminated before the balanced system formation. ${ }^{29}$ When two separated sources are present in the system, comparably, independent bell-shaped concentration gradients occur and an obvious spatial intersection is observed. At this interface region, a spontaneous molecule distribution overlapping play induces localized homogeneous elements mixing, and an integrated dual-cone spreading is pronounced. This molecule enriched interface describes an enforced molecular level collision at the diffusion fronts encountering boundary, which implies a regionally confined chemical mechanism.

To investigate the feasibility of molecular diffusion behavior controls in a pseudosolid phase, a PDMS-based model system with 7-amino-4-methylcoumarin as the diffusing target molecule and toluene as the carrying solvent was first testified. As shown in Figure 1A, 7-amino-4-methylcoumarin molecules diffuse from the edge of a liquid reservoir to enter the pseudosolid PDMS phase, where their traveling distance is dependent upon the system operation duration. This behavior can be quantified by defining a diffusion velocity parameter $Q$ which is equal to $\mathrm{d} x / \mathrm{d} t$, representing the diffusion front moving distance per time period. In a liquid or gas matrix environment, individual molecular movements are highly related to particle size and mass. This particle dimension dependent phenomenon is similarly observed when molecules with different molecular mass diffuse from a liquid phase to another solid related matrix, carried by the same solvent within a specific period of time (Figure 1B). A descending trend in diffusion front moving distance was observed, following the molecular mass order: 4-hydroxybenzoic acid <7-amino-4methylcoumarin $<$ terphenyl $<$ Nile red $<$ curcumin. This indicates an inversely proportional relationship between $Q$ and molecular mass as shown in the inset. Another important parameter in molecular diffusion is operation system temperature, which is related to the kinetic energy of a moving molecule. As shown in Figure 1C, an increasing diffusion front movement was consistently observed when the system temperature rose from 40 to $50,60,70$, and $80{ }^{\circ} \mathrm{C}$, individually. A proportional relationship between $Q$ and temperature is therefore obtained (in the inset), indicating an accelerating molecule transportation when a higher energy is provided in the environment.

Different from the spontaneous diffusion behavior in the liquid or gas phase, a carrying solvent is always required to achieve efficient analyte movements in a pseudosolid matrix. A variety of organic molecules have been investigated, where characteristics including cohesive energy density, hydrogen bonding capability, and molecular polarity were found to be dominating their entrance into PDMS networks. ${ }^{16}$ Under the circumstances of fixed analyte (7-amino-4-methylcoumarin) and system temperature $\left(20{ }^{\circ} \mathrm{C}\right)$ parameters, various solvent molecules including toluene, ethyl acetate, acetone, ethanol, methanol, and water were therefore selected to examine the overall molecular difference effects on the solvent-triggered PDMS entrance capability. Blue fluorescent 7-amino-4methylcoumarin was used to assess the solvent diffusion velocity in the pseudosolid system because of the transparency 
(A)

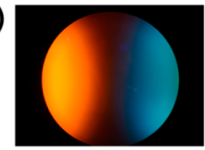

(B)

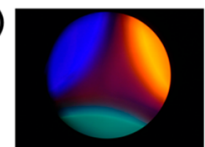

(C)

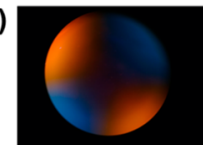

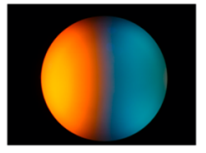
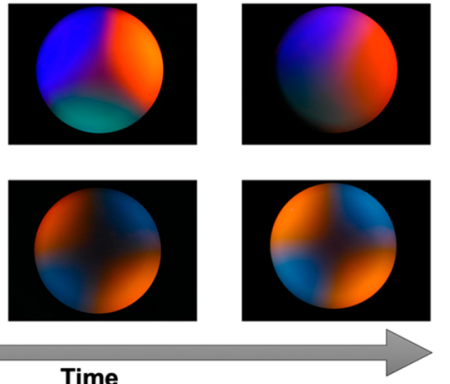

(D)

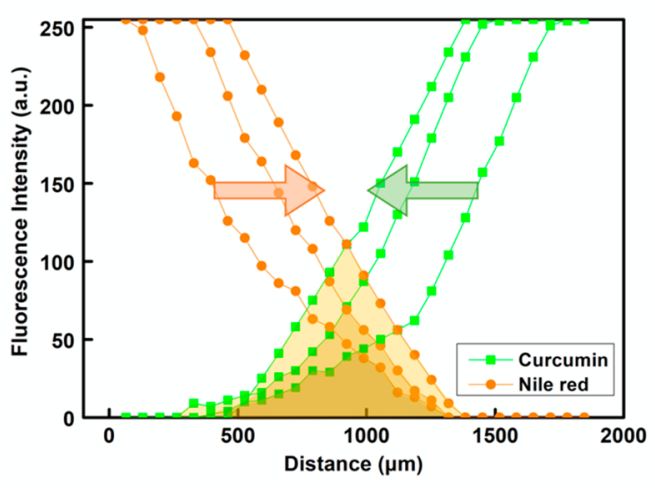

Figure 2. Diffusing and mixing of multiple fluorescent analytes with time from different sources monitored by a microscope. (A-C): $3000 \times 2300$ $\mu \mathrm{m}^{2}$ photo images showing a $3.14 \times 10^{6} \mu \mathrm{m}^{2}$ circular observation window in the middle. (A) Diffusion of curcumin (with green fluorescence) and Nile red (with orange fluorescence) from two opposite directions. (B) Diffusion of 7-amino-4-methylcoumarin (with blue fluorescence), Nile red (with orange fluorescence), and curcumin (with green fluorescence) from three independent sources. (C) Diffusion of curcumin (with green fluorescence) from two diagonal sources, with Nile red (with orange fluorescence) diffusing from the other adjacent two. (D) Image color analysis of A, where different colors (green represents curcumin and orange represents Nile red) are transferred into corresponding spatial intensity distribution. With the increase in diffusion duration, the overlapping area of two different color profiles rises, indicating a higher level of two components' mixing.

(A)

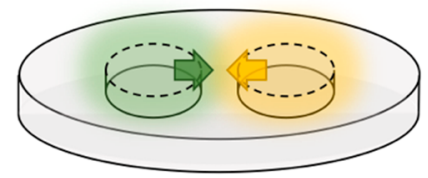

(C)

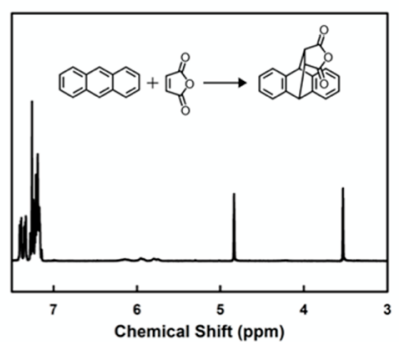

(B)

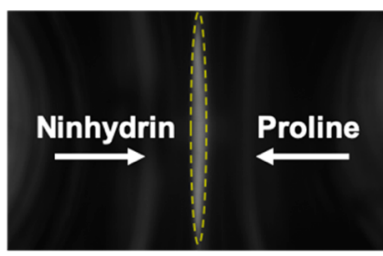

(D)

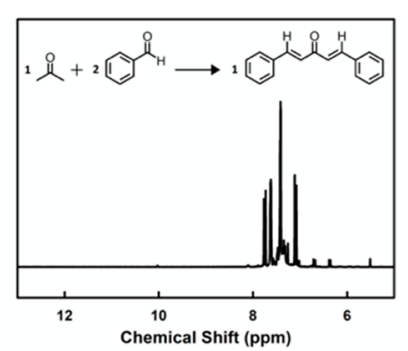

(E)

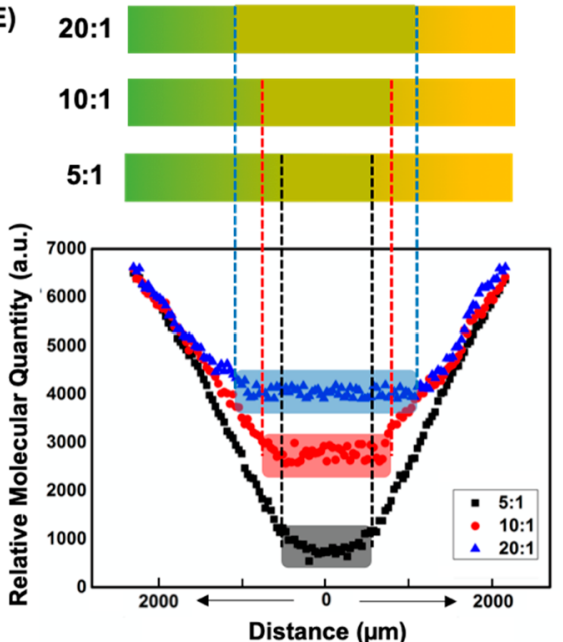

Figure 3. Demonstrations of using PDMS as a pseudosolid phase selective chemical reaction platform for providing straightforward experimental operation and high purity products. (A) Illustration of the PDMS-based diffusion-driven reaction device. Two isolated reservoirs are created and reactants are allowed to freely diffuse when the process initiates. The diffusion moving fronts deliver limited quantities of analytes, and chemical reactions can happen at the restricted encountering interface area efficiently. (B) The fluorescence image showing an opal region where diffusion fronts of ninhydrin and proline from two opposite directions overlap, indicating a successful ninhydrin test result in this design. (C) Diels-Alder reaction and (D) aldol condensation tests, where NMR spectra of chemical reaction products collected from PDMS slices at reaction regions are examined. Pure products without reactant residues indicate the advantageous property of this approach. (E) Identical Diels-Alder reactions are employed in PDMS devices prepared by different polymer base/curing agent ratios, including 5:1, 10:1, and 20:1. Different relative molecular quantity profiles represent network polymerization effects in this system.

of tested solvents. With the use of higher-polarity solvent molecules, a reducing diffusion front movement was observed (Figure 1D), following the order of toluene $(0.099)>$ ethyl acetate $(0.228)>$ acetone $(0.355)>$ ethanol $(0.654)>$ methanol $(0.762)>$ water $(1.000) .^{30}$ This observation points to a higher tendency of analyte entrance into PDMS networks when a less-polar carrying solvent was employed in the process, which follows an obvious inversely proportional trend. Interestingly, an apparent conversion point (ethanol in this case) is observed when the system is operated at $20^{\circ} \mathrm{C}$, and a nonsmooth $Q /$ relative polarity curve is obtained (Figure $1 \mathrm{E}$ ). It is believed that this negative bias from a predicted behavior is attributed to the activation energy requirement for the target analytes to enter pseudosolid phase PDMS by carrying solvents. $^{31}$ An elevated system temperature is known to provide higher kinetic energy to mobile molecules, ${ }^{32}$ and a smoothly fitted $Q /$ relative polarity curve is therefore expected. As shown in the Figure 1E inset, the ethanol molecule presents lower experimental $Q$ values toward the inversely proportional trend between $Q$ and solvent relative polarity. Meanwhile, higher temperatures deliver larger $Q$ values and better fitted inversely proportional tendencies are obtained, which should be relevant to the PDMS entrance activation energy. According to Fick's first law, the diffusion coefficient is proportional to 
(A)

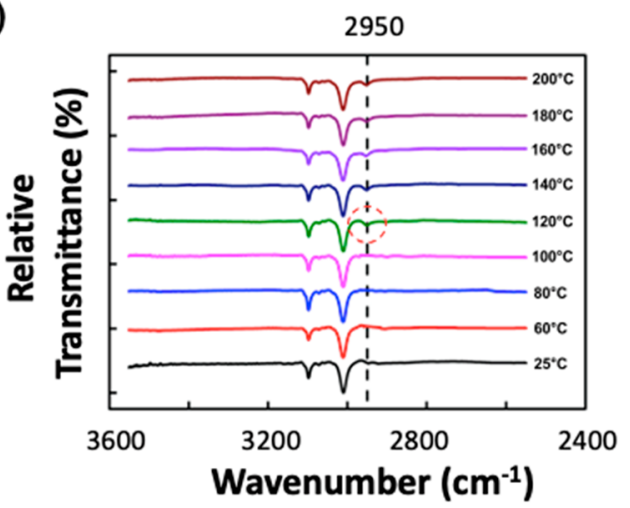

(B)

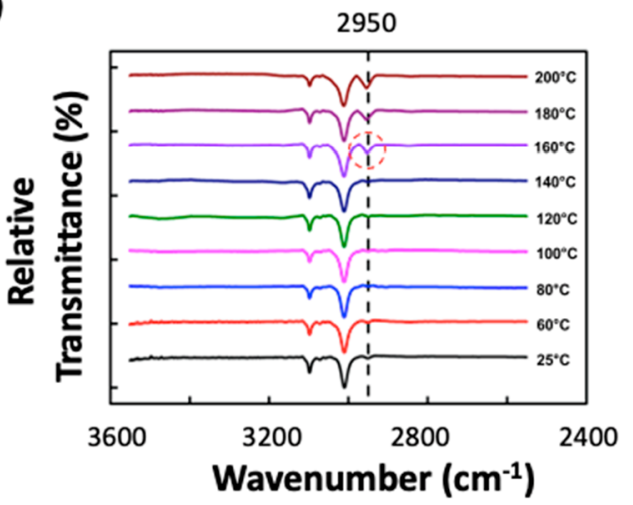

(C)

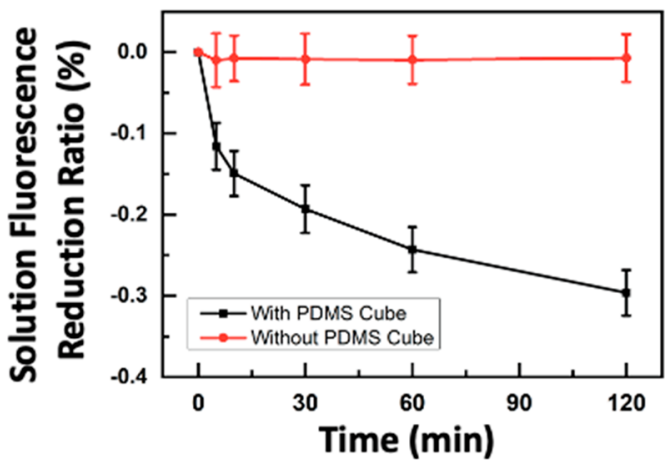

(D)

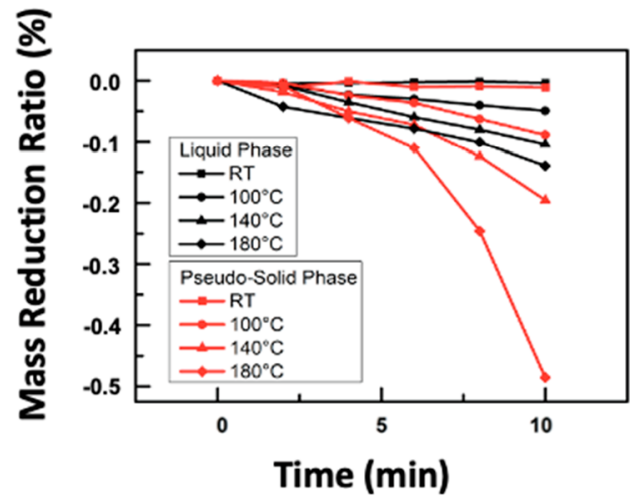

Figure 4. Investigation of the chemical reaction rate increase in the pseudosolid phase. (A, B): Identical representative Diels-Alder reactions operated (A) in the pseudosolid phase PDMS matrix and (B) in the liquid phase toluene system. A comparably lower temperature is required to initiate chemical reactions (marked by dash circles) in the pseudosolid phase. (C) Nile red solution fluorescence reduction rates with and without the presence of a PDMS cube in the solution. The reduction rate is defined as $\frac{F_{t}-F_{0}}{F_{0}} 100 \%$, where $F_{0}$ and $F_{t}$ denote the solution fluorescence intensity at initial and different time periods, respectively. (D) Solvent mass reduction rates in liquid and pseudosolid phases under different temperatures. The mass reduction rate is defined as $\frac{W_{t}-W_{0}}{W_{0}} 100 \%$, where $W_{0}$ and $W_{t}$ represent the weight at initial and different time periods, respectively.

the squared particle diffusion velocity and is dependent upon the system temperature. The activation energy of different solvents can therefore be calculated by the Arrhenius equation via slopes of the proportional relationship in a $\ln Q$ versus $1 / T$ plot (Figure S1). As summarized in Table S1, the highest PDMS entrance activation energy is required for ethanol solvent molecules. This result represents an inefficient energy for the solvent to overcome the interphases transportation energy barrier at $20^{\circ} \mathrm{C}$, which is consistent with the negative bias phenomenon observed in a $Q /$ relative polarity curve. With the rise of system temperature, consequently, this energy barrier can be overcome to reach higher $Q$ values, and a better inversely proportional $Q /$ relative polarity relationship is fulfilled.

Under the presence of a more sophisticated system, i.e., multiple initial sources, the organic solvent-triggered analyte molecular diffusion phenomenon can be relatively complicated. As demonstrated in Figure 2, two, three, and four source spots were used to reveal the conjugated fluorescent analyte interactions inside PDMS when introduced via molecular diffusion by the toluene solvent. This visual examination applied analytes, including curcumin and Nile red for the two sources test (Figure 2A); curcumin, 7-amino-4-methylcoumarin, and Nile red for the three sources test (Figure 2B); and curcumin with Nile red for the four sources test (Figure 2C). At the initial stage, the distinct and pure color fluorescence signal edge is found to belong to a specific molecule. Movements of fluorescent molecules driven by the organic carrying solvent with time result in expanding edge fronts and finally produce an overlapping area rendering a color mixing effect. These analytes mixing induced color fluctuation can also be quantified by a position-dependent color profile of each test, where a longer diffusion period is found to produce elongated and extensive color cohesion (Figure 2D).

Inspired by the aforementioned multicomponent mixing effect in a diffusion system, we envision a pseudosolid phasebased chemical reacting platform capable of providing straightforward experimental operation and high purity products. This design enables efficient reactions happening in a confined matrix, where individual reactants travel into a localized region via molecular diffusion carried out by mobile solvents, as depicted in Figure 3A. Because of the presence of molecular concentration gradients in a diffusion phenomenon, molecular moving fronts carrying a small number of reactants encounter at the limited boundary space. The continuous and limited supply of reactants facilitates effective molecular collision in the confined region, resulting in pronounced chemical process completion. Since movements of molecules in a pseudosolid matrix are spatially restricted, the directional guidance provided by carrying solvents avoids random transfer of both reactants and products. Furthermore, unreacted molecules cannot cross over the intermediate reaction zone 

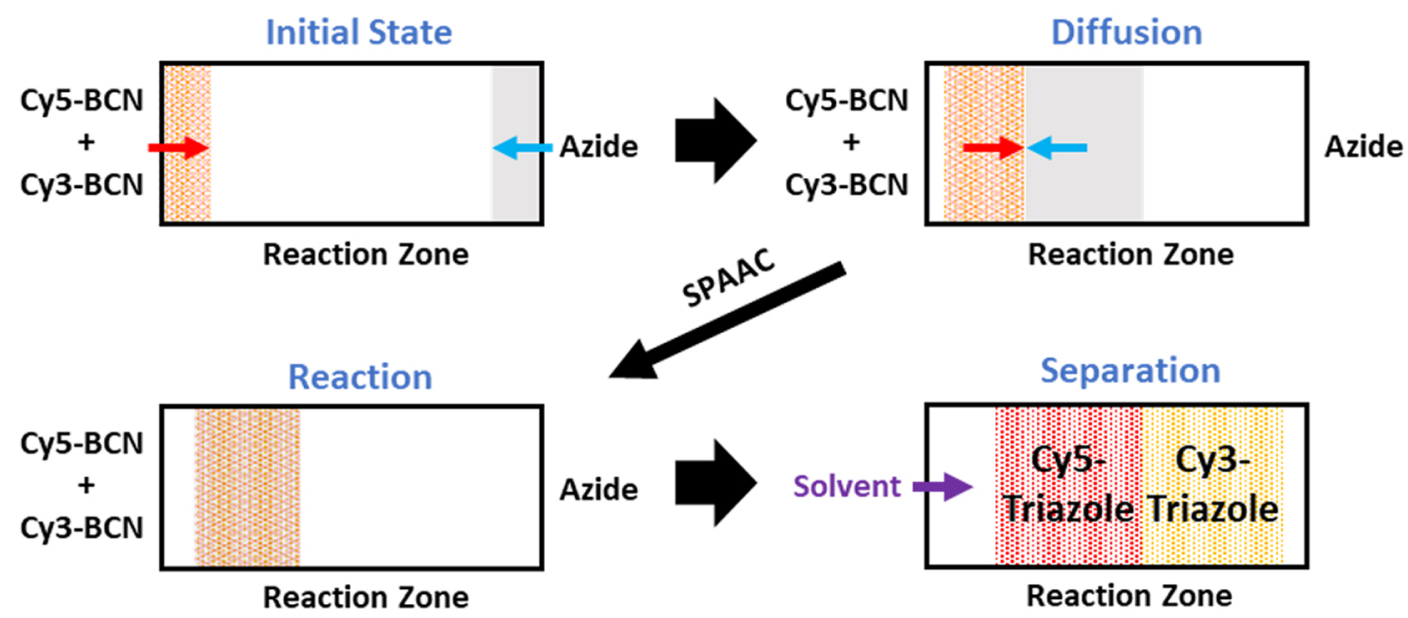

Cy5
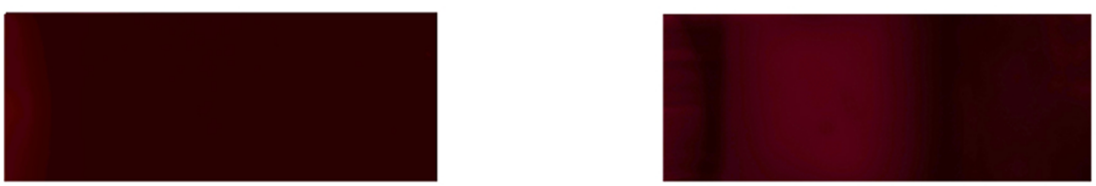

Cy3
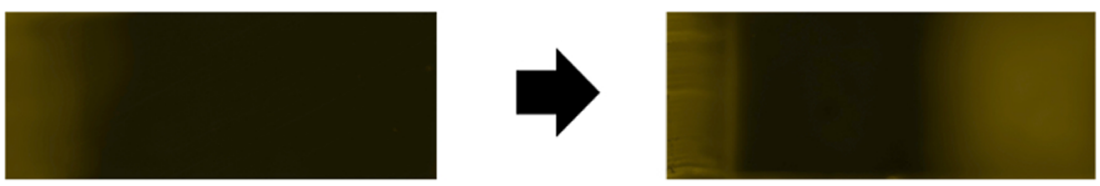

Merge
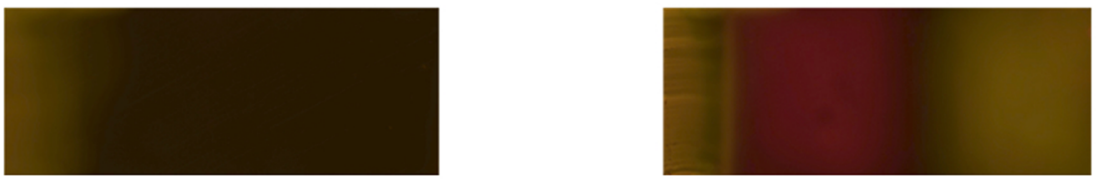

Figure 5. Achievements of effective and complicated multicomponent chemical reactions, with the simultaneous separation of final products in this diffusion-driven pseudosolid matrix-based chemical reaction platform. A strain-promoted azide-alkyne cycloaddition (SPAAC) process between fluorophore-tethered alkyne (Cy5-BCN and Cy3-BCN) and azide (azido-amine) is utilized as the model reaction. Both Cy5-BCN and Cy3-BCN are introduced into the pseudosolid PDMS system from the left-hand side, and azido-amine is brought in from the right. Monitored by a fluorescence microscope, images are collected from the red (Cy5, Excitation/Emission: $620 \pm 25 / 690 \pm 25 \mathrm{~nm}$ ) and the yellow (Cy3, Excitation/ Emission: $540 \pm 15 \mathrm{~nm} / 605 \pm 25 \mathrm{~nm}$ ) channels, and a merging of the two is also produced. As evidenced from the fluorescence images, the produced final products are simultaneously separated into two bands at the end of operation.

when entering this area because of the enforced reacting. Highpurity product generation and accumulation is therefore expected and should be allocated within a middle position in the pseudosolid matrix.

To obtain process produced pure substances, the pseudosolid phase reaction zone is cut out and dipped into solvents for direct matrix-trapped component extraction. For the convenience of experimental observation, a widely used ninhydrin test for amine moiety recognition is adopted to verify the described pure product generation in a pseudosolid matrix. ${ }^{33}$ As shown in Figure $3 \mathrm{~B}$, ninhydrin and proline are introduced from separate source spots, where the secondary amine in a proline structure should react with ninhydrin to produce colored species at the encountering interface of the moving fronts. The obvious product generation region marked by the dashed line in Figure $3 \mathrm{~B}$ indicates that the molecular diffusion in a confined space can not only provide efficient components mixing effects but also enforce chemical reactions to occur. Importantly, this phenomenon is extendable toward different reactions to achieve pure chemical production. In
Figure 3C, D, representative Diels-Alder reaction and aldol condensation, respectively, are tested under the same operation processes in this pseudosolid phase system. The generated products extracted from the PDMS matrix with no need of further purification are also found pure without reactant residues under NMR spectrum characterization. It is therefore concluded that diffusion fronts deliver limited quantities of molecules to initiate efficient chemical reactions happening in a confined region and yield high-quality products without excess precursors, whereas the slow movement of the analyte helps the separation of the product and the excess precursors. Unlike the high dilution method, where a desired chemical reaction is promoted through a low concentration reactant usage, high-quality products are produced in this operation due to the excess precursor prior segregation.

The molecular diffusion behavior in solid-related phases, however, always suffers from the property of slow movements, which hinders its practical application. Fortunately, this disadvantageous effect can be overcome by polymer network porosity adjustments in our operation. As demonstrated in 
Figure 3E, different PDMS polymerization recipes are tested including 5:1, 10:1, and 20:1 of polymer base/curing agent ratio. ${ }^{34}$ Under the same diffusion-driven Diels-Alder reaction operation mentioned in Figure 3C, relative molecular quantities of reactant species from two opposite entrance sides are all showing decreasing trends toward the central molecule encountering interfaces. It is interesting to note that steady reaction product quantity platforms presenting in the middle region follow the trend of 20:1 > 10:1 > 5:1, indicating more product generation in a less-polymerized matrix under the same experimental condition and time frame. Furthermore, wider reaction product platform distributions are also found following the same changing tendency, which is consistent with faster molecular movements in a looser polymer network. Both higher production results and faster reaction speeds observed here point to controllable and achievable efficient chemical reactions, which can resolve the conventional concerns in a solid-related phase system.

Despite the convenient design and high-quality product generation, a reaction rate increase is expected when chemical processes are operated in a pseudosolid phase system. This anticipation is based on the spatial limitation in polymer networks, where reactant molecules are confined within a specific space and effective intermolecular collision frequencies are raised. As depicted in Figure 4A, B, Diels-Alder reaction experiments in this pseudosolid phase and a liquid phase are performed in parallel, and their corresponding product generations is monitored by IR spectroscopy, respectively. For Diels-Alder reaction performed in the pseudosolid phase, the PDMS cube is preimmersed into the reactants containing toluene solution for $30 \mathrm{~min}$ before reaction initiation to eliminate slow diffusion factors during the process. Three characteristic peaks are found at 3050,3000, and $2950 \mathrm{~cm}^{-1}$, which are attributed to $\mathrm{C}-\mathrm{H}$ aromatics vibrational stretching, $\mathrm{C}-\mathrm{H}$ alkene vibrational stretching, and $\mathrm{C}-\mathrm{H}$ alkane vibrational stretching, respectively. ${ }^{35}$ Notably, both reactants and products give peaks at 3050 and $3000 \mathrm{~cm}^{-1}$, where the peak at $2950 \mathrm{~cm}^{-1}$ is the sole contribution from the final product and is selected as the monitoring target. Because of the heating operation in the Diels-Alder reaction, identical experiments under different temperatures are independently performed in a PDMS cube (Figure 4A) and in a liquid toluene solvent (Figure 4B). In these experiments, the main force of molecules mixing is diffusion-driven mass transfer rather than convection. It is obvious that under $3 \mathrm{~min}$ of reaction time, successful product generation starts from $120^{\circ} \mathrm{C}$ in a pseudosolid matrix, whereas a $160{ }^{\circ} \mathrm{C}$ operation is required in the liquid solvent environment. As the reaction still proceeds at $120^{\circ} \mathrm{C}$ but with a much lower rate (Figure S2), this phenomenon could be the result of a kinetic increase in reaction rate. Nonetheless, through calculating the activation energy values of this reaction in PDMS and in liquid toluene, it is found that the PDMS matrix indeed offers activation energy that is a magnitude lower in this process. Both effects are therefore believed to be sources of the observed interesting behavior in PDMS.

Two unique properties of the PDMS matrix have been hypothesized to further understand the mechanism behind the observation. First, hydrophobic molecules tend to diffuse into and be retained by PDMS, resulting in molecule accumulation. To verify this hypothesis, a PDMS piece is immersed in Nile red alcohol solution and the solution's fluorescence intensity is monitored. As evidenced in Figure 4C, the Nile red content in the solution decreases with time when the PDMS cube is present in the solution. On the contrary, the Nile red quantity in a solution stays constant without the PDMS piece. Second, the solvent evaporation rates in pseudosolid and pure liquid phases are assumed to be different. ${ }^{36}$ For comparison, the differences in solvent mass reduction between a PDMS cube saturated with ethanol and a pure ethanol solution are monitored under different temperatures (Figure 4D). The ethanol mass loss rate in PDMS is found to be much higher than in the liquid phase, presumably caused by the capillary effect at the PDMS/atmosphere interface. ${ }^{36}$ These two experiments reveal that the PDMS matrix can not only accumulate hydrophobic molecules from surrounding solutions but also facilitate volatile solvent evaporation. Both factors consequently provide a solute enriching phenomenon, which helps promote spatial limitation for more effective molecular collisions in the pseudosolid polymer networks.

Remarkably, the current strategy can boost more complicated chemical reaction operations and, in the meantime, commit the simultaneous separation of final products. As shown in demonstrative click reactions performed inside PDMS (Figure 5), two types of bicyclo[6.1.0] nonyne (BCN) tethered with different cyanine fluorescent dyes ( $\mathrm{Cy} 5$ and Cy3) are introduced from the left-hand side and an azide containing species is provided from the opposite end. During the molecular diffusion period, three individual reactants reach the central reaction zone and initiate the strain-promoted azide-alkyne cycloaddition (SPAAC) to convert precursors into a mixture of two fluorescent final products. Similar to the molecule quantity limiting phenomenon at diffusion fronts described above, reactants entering the confined boundary space undergo more complete reactions, and precursor residues are avoided. Under continuous supply of carrying solvents from the left-hand side, the mixture of two fluorescent products migrates inside pseudosolid PDMS networks in a chromatography-like fashion, and a simultaneous final product band separation is achieved at the end because of products' different affinities toward PDMS. This exciting result reveals an alternative strategy for perpetrating chemical reactions and delivers a potential tool for efficient product generation without the hassle of final treatments afterward.

\section{CONCLUSIONS}

In summary, an unconventional approach rendering chemical reaction facilitation and simultaneous final product separation properties is demonstrated. This technique adopts delicate molecular spatial controls as the driving force to deliver efficient chemical processes and to trigger a traceable functioning mechanism. The introduced strategy utilizes a pseudosolid environment to achieve molecule quantity regulation and moving direction guidance while overcoming regular diffusion behavior and velocity concerns in a solidrelated matrix system. For investigating fundamental properties of the molecular diffusion phenomenon in a pseudosolid matrix, crucial factors including molecular size effects, system temperature influences, and carrier solvent varieties are investigated. Compared to considering Fick's diffusion law parameters in a solely gas or liquid environment, executing chemical operations in a pseudosolid phase elaborates interphase molecular movement tracing and is highly dependent upon the matrix entrance capability of carrying solvents. This factor settles an energy barrier of matter transportation between two phases, where an elevated system temperature provides higher kinetic energy for molecules to fulfill the 
minimum pseudosolid phase entrance requirement. When molecules successfully diffuse into a solid matrix, the spatial concentration difference occurs and the formation of a continuous descending gradient distribution delivers minimized molecule quantities at its moving front. With directional controls of these fronts in a sophisticated system, delicate molecular transportation and effective components mixing are achieved. Taking advantage of this straightforward operation, chemical reactions can be triggered at the overlapping area of these diffusion fronts. Meanwhile, this area creates a molecular collision hot spot by limiting a certain number of molecules in a confined region. High-quality final products with minimized reactant residues are therefore obtained at the end of the operation because of the enforced effective molecular collisions. Representative chemical reactions including ninhydrin test, Diels-Alder reaction, and aldol condensation are testified, which all point to similar pure product generation via this spatial limitation-based approach. In addition to the advantageous properties described above, facilitated chemical reaction rate and lowered activation energy are found in this pseudosolid system compared to a liquid-based operation, which is believed to be induced by enhancing effective molecular collisions. Remarkably, applying multiple components for a complicated reaction under the same principle can not only reach correct chemistry but also separate final products simultaneously in the process. Because of the end product trapping property inside a polymerized matrix, the target portion can simply be sliced to obtain desired substances. Based on these fascinating characters and findings, we foresee this unique pseudosolid matrix operation system to be further applied in fine chemistry and other extending molecular control phenomena.

\section{EXPERIMENTAL SECTION}

\section{Materials and Reagents}

Anthracene and maleic anhydride were purchased from Alfa Aesar (Lancashire, UK). Proline and ninhydrin were purchased from Tokyo Chemical Industry (Tokyo, Japan). Potassium bromide (KBr) IR grade, Nile red, 4-hydroxybenzoic acid, 7-amino-4-methylcoumarin, terphenyl, and curcumin were purchased from Sigma-Aldrich (St. Louis, MO, USA). Sodium hydroxide was purchased from SHOWA (Tokyo, Japan). Toluene, acetone, hexane, and ethanol were purchased from Echo Chemical (Taipei, Taiwan). Acetic acid was purchased from Aencore (Victoria, Australia). Ethyl acetate was purchased from Seedchem (Melbourne, Australia). These reagents were used as supplied unless otherwise stated. Ultrapure water $(>18.2$ $\mathrm{M} \Omega \mathrm{cm}^{-1}$ ) generated from an ELGA PURELAB classic system (Taipei, Taiwan) was used in all experiments. SYLGARD 184 silicone elastomer base and curing agent were purchased from Dow Corning Corp. (Midland, MI, USA). Cy5 and Cy3 tethered bicyclo[6.1.0]nonyne $(\mathrm{BCN})$ and 2-[2-(2-azidoethoxy)ethoxy]ethanamine click reagents were prepared by following a reported method and confirmed by NMR spectroscopy. ${ }^{37,38}$

\section{Instruments and Equipment}

Fourier transform IR (FTIR) spectra were obtained from Thermo Scientific Nicolet iS5 FTIR spectrometer (Waltham, MA, USA) with $\mathrm{KBr}$ pellets. Photos and microscope images were captured with Leica D-LUX 6 digital camera and Zeiss epifluorescence microscope (Axio Imager. M2, Carl Zeiss microscopy, Jena, Germany), respectively. Hand-held UV lamp (6 W, UVGL-58) equipped with 254 and 365 $\mathrm{nm}$ of irradiation was purchased from Science Company (Denver, CO, USA). NMR spectra were acquired from Varian $400 \mathrm{MHz}-\mathrm{NMR}$ spectrometer (Palo Alto, CA, USA), and ${ }^{1} \mathrm{H}$ NMR spectra were performed in the $\mathrm{CDCl}_{3}$ solvent. UV-vis absorption spectra were acquired from Thermo Scientific Evolution UV-220 spectrometer
(Waltham, MA, USA). Precoated silica gel 60 F254 high-performance thin-layer chromatography (TLC) plates were purchased from Merck (Darmstadt, Germany). Electrospray ionization (ESI) mass spectra were obtained from Bruker micrOTOF-QII mass spectrometer (Billerica, MA, USA).

\section{Preparation of PDMS Cubes}

PDMS cubes were prepared by thoroughly mixing the SYLGARD 184 silicone elastomer base and curing agent in a mass ratio of 10:1 and vacuumed to remove gas bubbles. After a clear and transparent mixture solution was obtained, the liquid PDMS was poured into a cleaned mold and cured at $100{ }^{\circ} \mathrm{C}$ for $1.5 \mathrm{~h}$ with a subsequent $60{ }^{\circ} \mathrm{C}$ overnight baking. The solidified PDMS pieces can be cut into desired dimensions, and a fixed 10:1 elastomer base/curing agent ratio was employed throughout the experiment unless otherwise stated.

\section{Molecular Diffusion Behavior Monitoring in PDMS}

To monitor molecular diffusion behavior and investigate important affecting factors in the system, $0.01 \mathrm{mM}$ (in toluene) of target analytes including 4-hydroxybenzoic acid, 7-amino-4-methylcoumarin, terphenyl, Nile red, and curcumin were applied. For the system temperature effect study, 7-amino-4-methylcoumarin was used as the model molecule and the system was operated at $40,50,60,70$, and 80 ${ }^{\circ} \mathrm{C}$, respectively. Solvent effect studies were performed by preparing $0.01 \mathrm{mM}$ 7-amino-4-methylcoumarin in toluene, ethyl acetate, acetone, ethanol, methanol, and water, respectively. For quantification of these diffusion processes, fluorescent microscope images were analyzed by the MATLAB program.

\section{Non-Reactive Multi-Component Mixing Effects}

7-Amino-4-methylcoumarin, Nile red, and curcumin were prepared in toluene with $0.01 \mathrm{mM}$ for this study. A PDMS cube designed with different numbers of liquid reservoirs was used to hold analyte liquids, and molecular diffusion was observed after the introduction of liquids. Photos were taken under $365 \mathrm{~nm}$ of UV excitation, and the obtained images were analyzed by the ImageJ program.

\section{Efficient Chemical Reactions in PDMS}

For reaction tests in the pseudosolid phase, a PDMS cube with two reservoirs was prepared to provide the double diffusion source system. In the ninhydrin test, $2 \mathrm{~mL}$ of ninhydrin $(0.08 \mathrm{~g}$ in $20 \mathrm{~mL}$ ethanol) and $2 \mathrm{~mL}$ of proline $(0.04 \mathrm{~g}$ in $20 \mathrm{~mL}$ of ethanol with $1 \mathrm{~mL}$ of $10 \mathrm{mM}$ acetic acid) were separately introduced to individual reservoirs, and diffusion was allowed to occur under the ambient condition. After 30 min, the produced colored region can be observed by the naked eye and recorded by a digital camera.

In the Diels-Alder reaction test, $5 \mathrm{~mL}$ of anthracene $(0.04 \mathrm{M}$, in toluene) and $5 \mathrm{~mL}$ of maleic anhydride $(0.04 \mathrm{M}$, in toluene) were introduced into two separated reservoirs. After $60 \mathrm{~min}$ of diffusion induced mixing, the reactant containing PDMS cube was heated at $200{ }^{\circ} \mathrm{C}$ for $30 \mathrm{~min}$. This PDMS cube was thereafter dipped into 10 $\mathrm{mL}$ of toluene with $30 \mathrm{~min}$ of sonication for product extraction. The solution was then collected and tested by NMR for qualitative analysis.

In the aldol condensation reaction test, $5 \mathrm{~mL}$ of benzaldehyde $(0.05 \mathrm{M}$, in ethanol) and $5 \mathrm{~mL}$ of acetone $(0.05 \mathrm{M}$, in ethanol with 1 $\mathrm{g}$ of sodium hydroxide) were introduced into PDMS reservoirs separately. After $60 \mathrm{~min}$ of diffusion induced mixing and room temperature reaction, the PDMS cube was dipped into $10 \mathrm{~mL}$ of ethanol with $30 \mathrm{~min}$ of sonication for product extraction. The solution was then collected and tested by NMR for qualitative analysis.

Reaction Rates Comparison between Pseudo-Solid and Liquid Phase Operations

The Diels-Alder reaction test was employed to understand the reaction rate difference between pseudosolid and liquid phase operations under the same condition. Twenty-five milliliters of anthracene $(0.04 \mathrm{M}$, in toluene $)$ and $25 \mathrm{~mL}$ of maleic anhydride $(0.04$ $\mathrm{M}$, in toluene) were first mixed and stirred for $5 \mathrm{~min}$. A $1 \times 1 \times 0.5$ $\mathrm{cm}^{3}$ PDMS cube was thereafter immersed into $2 \mathrm{~mL}$ of the mixture solution for $30 \mathrm{~min}$ of absorption to allow complete intake of reactants into the PDMS matrix, followed by $\mathrm{N}_{2}$ drying and 3 min of 
heating on preheated hot plate at different temperatures. After heating, the PDMS cubes were then soaked into $3 \mathrm{~mL}$ of toluene for 2 $\mathrm{h}$ of product extraction. For comparison, the same reactions were performed in liquid toluene without the use of PDMS. This experiment design has eliminated the molecule diffusion rate difference effect in two phases, and can provide a parallel comparison of reaction rates. To identify reactants and products after reaction operation in both phases, TLC plate spotting developments (in 2:8 ethyl acetate/hexane) were performed and monitored under $254 \mathrm{~nm}$ of UV light. FTIR characterization using $\mathrm{KBr}$ pellets was also employed for final product confirmation and comparison.

\section{Reaction Rate Facilitation Mechanism Understanding}

To investigate the accumulation of Nile red molecules into the PDMS matrix, a $1 \times 1 \times 0.5 \mathrm{~cm}^{3}$ PDMS piece was submerged into a 0.0025 $\mathrm{M}$ Nile red ethanol solution. The solution's fluorescence emission at $630 \mathrm{~nm}$ was monitored under different time periods and compared with another Nile red ethanol solution without PDMS immersion. For solvent evaporation behavior comparison tests, the weight loss of an ethanol-saturated PDMS (presoaked in ethanol for $30 \mathrm{~min}$ ) and an independent ethanol solution was monitored under different temperatures.

\section{Chemical Reactions with Simultaneous Product Separation}

For the convenience of observation under a fluorescence microscope, a $0.1 \times 0.2 \times 0.5 \mathrm{~cm}^{3}$ PDMS piece was used in this test. Two ends of the PDMS piece were separately introduced with the click reagent mixture (0.01 M of Cy5-BCN and $0.01 \mathrm{M}$ of $\mathrm{Cy} 3-\mathrm{BCN}$ in toluene) and azido-amine $(0.01 \mathrm{M}$ in acetone) solutions, respectively. Two hours of free diffusion time was allowed for complete alkyne-azide click reaction, after which more toluene was added from the click reagent side to separate the products, and the distribution of products was monitored by a fluorescence microscope. (Note: The BCN used here can react with azides to form SPAAC click reaction products under ambient conditions without the need of metal catalyst..$^{39}$ ) Because the generated products were trapped inside polymerized PDMS after the reaction, slicing of different PDMS portions can simply separate individual components. For the confirmation of the produced compounds, chemicals inside PDMS slices were extracted by toluene and characterized by FTIR after rotary evaporative removal of solvents. The disappearance of the azide characteristic peak at 2100 $\mathrm{cm}^{-1}$ reveals obvious active moiety consumption in this operation, which is consistent with the additional mass spectrometry results, showing successful occurrence of alkyne-azide click reaction.

\section{ASSOCIATED CONTENT}

\section{Supporting Information}

The Supporting Information is available free of charge at https://pubs.acs.org/doi/10.1021/jacsau.1c00202.

Figure $S 1$, relationship between $\ln Q$ and the reciprocal of system temperature; Table S1, calculation of PDMS entrance activation energy by Arrhenius equation; Figure S2, IR spectra of representative Diels-Alder reaction in the liquid phase under different conditions (PDF)

\section{AUTHOR INFORMATION}

Corresponding Author

Wei-Ssu Liao - Department of Chemistry, National Taiwan University, Taipei 10617, Taiwan; ㅇo이.org/0000-00025619-4997; Email: wsliaochem@ntu.edu.tw

\section{Authors}

Shin Yi - Department of Chemistry, National Taiwan University, Taipei 10617, Taiwan

Jia-Syuan Chen - Department of Chemistry, National Taiwan University, Taipei 10617, Taiwan
Chang-Ming Wang - Department of Chemistry, National Taiwan University, Taipei 10617, Taiwan

Complete contact information is available at:

https://pubs.acs.org/10.1021/jacsau.1c00202

\section{Author Contributions}

${ }^{\dagger}$ S.Y. and J.-S.C. contributed equally

Notes

The authors declare no competing financial interest.

\section{ACKNOWLEDGMENTS}

This work was supported by the Taiwan Ministry of Science and Technology (MOST 108-2628-M-002-011-MY3). The authors thank Prof. Lee-Chiang Lo's group at National Taiwan University for supplement of organic compounds. The authors also thank Prof. Paul Cremer at The Pennsylvania State University for useful discussion of this work.

\section{REFERENCES}

(1) Wisniak, J.; Thomas Graham, Ii. Contributions to Diffusion of Gases and Liquids, Colloids, Dialysis, and Osmosis. Educ. Quim. 2013, $24,506-515$.

(2) Graham, T. X. Liquid Diffusion Applied to Analysis. Philos. Trans. R. Soc. London 1861, 151, 183-224.

(3) Fick, A. Ueber Diffusion. Ann. Phys. 1855, 170, 59-86.

(4) Paul, A.; Laurila, T.; Vuorinen, V.; Divinski, S. V. Thermodynamics, Diffusion and the Kirkendall Effect in Solids; Springer, 2014; pp 2-84.

(5) Miller, C. C. The Stokes-Einstein Law for Diffusion in Solution. Proc. R. Soc. London, Ser. A 1924, 106, 724-749.

(6) Young, M.; Carroad, P.; Bell, R. Estimation of Diffusion Coefficients of Proteins. Biotechnol. Bioeng. 1980, 22, 947-955.

(7) Liu, J.; Cao, D.; Zhang, L. Molecular Dynamics Study on Nanoparticle Diffusion in Polymer Melts: A Test of the StokesEinstein Law. J. Phys. Chem. C 2008, 112, 6653-6661.

(8) Wang, Z.; Kim, M.-C.; Marquez, M.; Thorsen, T. High-Density Microfluidic Arrays for Cell Cytotoxicity Analysis. Lab Chip 2007, 7, $740-745$.

(9) Zhou, J.; Ellis, A. V.; Voelcker, N. H. Recent Developments in Pdms Surface Modification for Microfluidic Devices. Electrophoresis 2010, 31, 2-16.

(10) Rumens, C. V.; Ziai, M. A.; Belsey, K. E.; Batchelor, J. C.; Holder, S. J. Swelling of Pdms Networks in Solvent Vapours; Applications for Passive Rfid Wireless Sensors. J. Mater. Chem. C 2015, 3, 10091-10098.

(11) Wawrzyniak, P.; Rogacki, G.; Pruba, J.; Bartczak, Z. Diffusion of Ethanol-Carbon Dioxide in Silica Gel. J. Non-Cryst. Solids 1998, 225, 86-90.

(12) Persson, L. B.; Morrison, G. M.; Friemann, J.-U.; Kingston, J.; Mills, G.; Greenwood, R. Diffusional Behaviour of Metals in a Passive Sampling System for Monitoring Aquatic Pollution. J. Environ. Monit. 2001, 3, 639-645.

(13) Si, G.; Yang, W.; Bi, S.; Luo, C.; Ouyang, Q. A Parallel Diffusion-Based Microfluidic Device for Bacterial Chemotaxis Analysis. Lab Chip 2012, 12, 1389-1394.

(14) Regehr, K. J.; Domenech, M.; Koepsel, J. T.; Carver, K. C.; Ellison-Zelski, S. J.; Murphy, W. L.; Schuler, L. A.; Alarid, E. T.; Beebe, D. J. Biological Implications of Polydimethylsiloxane-Based Microfluidic Cell Culture. Lab Chip 2009, 9, 2132-2139.

(15) Toepke, M. W.; Beebe, D. J. Pdms Absorption of Small Molecules and Consequences in Microfluidic Applications. Lab Chip 2006, 6, 1484-1486.

(16) Lee, J. N.; Park, C.; Whitesides, G. M. Solvent Compatibility of Poly (Dimethylsiloxane)-Based Microfluidic Devices. Anal. Chem. 2003, 75, 6544-6554. 
(17) Hu, S.; Ren, X.; Bachman, M.; Sims, C. E.; Li, G. P.; Allbritton, N. L. Surface-Directed, Graft Polymerization within Microfluidic Channels. Anal. Chem. 2004, 76, 1865-1870.

(18) Kim, B.-Y.; Hong, L.-Y.; Chung, Y.-M.; Kim, D.-P.; Lee, C.-S. Solvent-Resistant Pdms Microfluidic Devices with Hybrid Inorganic/ Organic Polymer Coatings. Adv. Funct. Mater. 2009, 19, 3796-3803.

(19) Lee, D.; Yang, S. Surface Modification of Pdms by Atmospheric-Pressure Plasma-Enhanced Chemical Vapor Deposition and Analysis of Long-Lasting Surface Hydrophilicity. Sens. Actuators, B 2012, 162, 425-434.

(20) Bax, D. V.; Kondyurin, A.; Waterhouse, A.; McKenzie, D. R.; Weiss, A. S.; Bilek, M. M. M. Surface Plasma Modification and Tropoelastin Coating of a Polyurethane Co-Polymer for Enhanced Cell Attachment and Reduced Thrombogenicity. Biomaterials 2014, $35,6797-6809$.

(21) Gevers, L. E. M.; Vankelecom, I. F. J.; Jacobs, P. A. SolventResistant Nanofiltration with Filled Polydimethylsiloxane (Pdms) Membranes. J. Membr. Sci. 2006, 278, 199-204.

(22) Rusina, T. P.; Smedes, F.; Klanova, J. Diffusion Coefficients of Polychlorinated Biphenyls and Polycyclic Aromatic Hydrocarbons in Polydimethylsiloxane and Low-Density Polyethylene Polymers. J. Appl. Polym. Sci. 2010, 116, 1803-1810.

(23) Karlsson, O.; Stubbs, J.; Karlsson, L.; Sundberg, D. Estimating Diffusion Coefficients for Small Molecules in Polymers and Polymer Solutions. Polymer 2001, 42, 4915-4923.

(24) Seethapathy, S.; Gorecki, T. Applications of Polydimethylsiloxane in Analytical Chemistry: A Review. Anal. Chim. Acta 2012, 750, $48-62$.

(25) Choi, S.-J.; Kwon, T.-H.; Im, H.; Moon, D.-I.; Baek, D. J.; Seol, M.-L.; Duarte, J. P.; Choi, Y.-K. A Polydimethylsiloxane (PDMS) Sponge for the Selective Absorption of Oil from Water. ACS Appl. Mater. Interfaces 2011, 3, 4552-4556.

(26) Adiraj Iyer, M.; Eddington, D. T. Storing and Releasing Rhodamine as a Model Hydrophobic Compound in Polydimethylsiloxane Microfluidic Devices. Lab Chip 2019, 19, 574-579.

(27) Kim, J.; Conway, A.; Chauhan, A. Extended Delivery of Ophthalmic Drugs by Silicone Hydrogel Contact Lenses. Biomaterials 2008, 29, 2259-2269.

(28) Philibert, J. One and a Half Century of Diffusion: Fick, Einstein, before and Beyond. Diffusion Fundamentals 2005, 2, 1.11.10 .

(29) Qian, H. Thermodynamics of the General Diffusion Process: Equilibrium Supercurrent and Nonequilibrium Driven Circulation with Dissipation. Eur. Phys. J.: Spec. Top. 2015, 224, 781-799.

(30) Reichardt, C.; Welton, T. Solvents and Solvent Effects in Organic Chemistry; John Wiley \& Sons, 2011; pp 425-490.

(31) Hayat, T.; Ullah, I.; Waqas, M.; Alsaedi, A. Attributes of Activation Energy and Exponential Based Heat Source in Flow of Carreau Fluid with Cross-Diffusion Effects. J. Non-Equilib. Thermodyn. 2019, 44, 203-213.

(32) Atkins, P.; De Paula, J. Physical Chemistry for the Life Sciences; Oxford University Press, 2011; pp 21-134.

(33) Ruhemann, S. Ccxii.-Triketohydrindene Hydrate. J. Chem. Soc., Trans. 1910, 97, 2025-2031.

(34) Ojuroye, O.; Torah, R.; Beeby, S. Modified PDMS Packaging of Sensory E-Textile Circuit Microsystems for Improved Robustness with Washing. Microsyst. Technol. 2018, 25, 1-18.

(35) Gouda, M. A.; Berghot, M. A.; Elattar, K. M.; Abd El Galil, M. K. Chemistry of Dibenzobarallene. Turk. J. Chem. 2011, 35, 663-697.

(36) Sharma, S.; Debenedetti, P. G. Evaporation Rate of Water in Hydrophobic Confinement. Proc. Natl. Acad. Sci. U. S. A. 2012, 109, 4365-4370.

(37) Gential, G. P.; Ho, N. I.; Chiodo, F.; Meeuwenoord, N.; Ossendorp, F.; Overkleeft, H. S.; van der Marel, G. A.; Filippov, D. V. Synthesis and Evaluation of Fluorescent Pam3cys Peptide Conjugates. Bioorg. Med. Chem. Lett. 2016, 26, 3641-3645.

(38) Ren, X.; El-Sagheer, A. H.; Brown, T. Azide and TransCyclooctene Dutps: Incorporation into DNA Probes and Fluorescent Click-Labelling. Analyst 2015, 140, 2671-2678.
(39) Dadfar, S. M. M.; Sekula-Neuner, S.; Bog, U.; Trouillet, V.; Hirtz, M. Site-Specific Surface Functionalization Via Microchannel Cantilever Spotting (Microcs): Comparison between Azide-Alkyne and Thiol-Alkyne Click Chemistry Reactions. Small 2018, 14, 1800131. 